\title{
Estratégias Metodológicas de Investigação da Resiliência em Lésbicas, Gays e Bissexuais (LGBs): Revisão Integrativa de Literatura
}

\author{
Aline Nogueira de Lira*, 1 \\ Orcid.org/0000-0002-7503-3742 \\ Normanda Araujo de Morais ${ }^{1}$ \\ Orcid.org/0000-0003-3156-4688
}

${ }^{1}$ Universidade de Fortaleza, Fortaleza, CE, Brasil

\begin{abstract}
Resumo
Pela revisão integrativa de literatura, este artigo buscou analisar as estratégias metodológicas para investigação da resiliência nos estudos empíricos com pessoas lésbicas, gays e bissexuais (LGBs). As buscas foram realizadas nas bases SciELO, PePSIC, LILACS, Index Psi, PsycINFO e PUBMED, até o ano de 2015. Para nortear e sistematizar a execução da revisão seguiu-se as diretrizes enunciadas no protocolo PRISMA e após a consideração dos critérios de inclusão e exclusão, foram identificados e analisados na íntegra 31 artigos. Identificou-se estudos com foco nas variáveis, com foco nas pessoas e os que incluem simultaneamente as variáveis e as pessoas. Apesar dos avanços conceituais e metodológicos acerca da resiliência e a população LGB, algumas controvérsias ainda são observadas, comprometendo a qualidade da avaliação desse construto. A medição e/ou compreensão da resiliência não é uma tarefa simplista, mas passa pela observação e diálogo entre os fatores de risco, de proteção e de adaptação positiva, além de envolver dialeticamente os diferentes níveis e contextos envolvidos: individual, familiar, comunitário e cultural. A inconsistência na operacionalização desses fatores pode prejudicar a generalização e a compreensão dos resultados empíricos.
\end{abstract}

Palavras-chave: Resiliência, LGB, método, revisão integrativa.

\section{Methodological Strategies for Resilience Research in Lesbians, Gays and Bisexuals (LGBs): Integrative Review of the Literature}

\begin{abstract}
Through the integrative literature review, this article aimed to analyze the methodological strategies for resilience research in empirical studies with lesbians, gays and bisexuals (LGBs). The searches were carried out at SciELO, PePSIC, LILACS, Index Psi, PsycINFO e PUBMED, until the year 2015. In order to guide and systematize the undergoing of the review, the guidelines laid down in the Protocol

* Endereço para correspondência: Universidade de Fortaleza, Avenida Washington Soares, 1321, Edson Queiroz, Fortaleza, CE, Brasil 60811 905. Caixa-postal: 1258. E-mail: aline.lira09@hotmail.com e normandaaraujo@, gmail.com

Apoio: Coordenação de Aperfeiçoamento de Pessoal de Nível Superior (CAPES); Conselho Nacional de Desenvolvimento Científico e Tecnológico (CNPq).
\end{abstract}


PRISMA were followed and after consideration of the inclusion and exclusion criteria, 31 articles were identified and analyzed in full. Studies focused only on variables, with focus only on people and including both variables and people were identified. Despite the conceptual and methodological advances about the resilience and the LGB population, some controversies are still observed, compromising the quality of evaluation of this construct. The measuring and/or understanding of resilience is not a simplistic task. It passes through observation and dialogue among risk factors, protection factors and positive adaptation factors, as well as dialectically relating to different levels and contexts involved such as individual, family, community and culture. The inconsistency in the operationalization of these factors can impair the generalization and the understanding of the empirical results.

Keywords: Resilience, LGB, method, integrative review.

\section{Estrategias Metodológicas de Investigación en Resiliencia en Lesbianas, Gays y Bisexuales (LGBs): Revisión Integrativa de la Literatura}

\section{Resumen}

Por la revisión integrativa de literatura, este artículo analizó las estrategias metodológicas para la investigación de la resiliencia en los estudios empíricos con lesbianas, gays y bisexuales (LGBs). La búsqueda fue realizada en las bases SciELO, PePSIC, LIIACS, Index Psi, PscycINFO y PUBMED, hasta el año de 2015. Para sistematiza la revisión de literatura, seguimos las directrices enunciadas en el protocolo PRISMA y luego de la consideración de los criterios de inclusión y exclusión, fueron identificados y analizados íntegramente 31 artículos. Se identificaron estudios con foco en las variables, en las personas y aquellos que incluyen a la vez las variables y las personas. A pesar de los avances conceptuales y metodológicos acerca de la resiliencia y de la población LGB, aspectos controversiales fueron observados, lo que comprometió la calidad de la evaluación del constructo. La medición y/o la comprensión de la resiliencia no es una tarea sencilla, pero pasa por la evaluación y el diálogo entre los factores de riesgo, de protección y de adaptación positiva, además de envolver dialécticamente diferentes niveles y contextos: individual, familiar, comunitario y cultural. La inconsistencia en la operacionalización de estos factores puede perjudicar la generalización y la comprensión de los resultados empíricos. Palabras-clave: Resiliencia; LGB; método, revisión integrativa.

Palabras clave: Resiliencia, LGB, método, revisión integrativa.

Crescente atenção tem sido dada aos processos de resiliência vivenciados por lésbicas, gays e bissexuais (LGBs), especialmente no cenário internacional. Para além do contexto de risco e vulnerabilidades que as minorias sexuais enfrentam cotidianamente em seus espaços sociais e familiares, sobretudo advindo do preconceito homofóbico, estudos recentes têm destacado a capacidade de enfrentamento e superação dessa população, enfatizando as estratégias individuais, familiares e comunitárias encontradas que as possibilitam prosperar e apresentar bons resultados desenvolvimentais (Lira \& Morais, 2017; Lyons, 2015). Desse modo, nem todas as pes- soas LGBs sucumbem às adversidades. Muitas delas, envolvidas em processos de resiliência, se fortalecem diante do estresse e sobressaem com mais recursos para levarem adiante os seus projetos de vida.

A resiliência é conceituada como a capacidade dinâmica e processual de gerir com êxito e se adaptar com sucesso às circunstância adversas da vida (Luthar, Cicchetti, \& Becker, 2000). A partir dessa definição, duas dimensões distintas compõem esse constructo: (1) a presença de situações e/ou contextos de adversidade significativos, identificados a partir da observação dos fatores de risco e; (2) a compreensão da adapta- 
ção positiva, ou seja, a existência de um conjunto de fatores e sistemas que contribuem para um processo dinâmico e interativo que aumentam a chance de resistir à adversidade (Luthar et al., 2000; Masten \& Monn, 2015).

Embora se constate uma relativa expansão da literatura sobre resiliência da população LGB, verifica-se que esses estudos encontram-se em estágios iniciais e que tanto a sua definição, quanto a sua mensuração/compreensão ainda representam um desafio (Colpitts \& Gahagan, 2016; Lyons, 2015). Primeiramente, os desafios se colocam pela falta de homogeneidade na definição da resiliência, que ora é definida como um traço, centrada sobretudo nas características individuais; ora como um processo dinâmico, o qual envolve a complexa relação entre fatores de risco, de proteção e adaptação positiva. Este estudo parte, portanto, dessa última concepção; segundo a qual a resiliência não se restringe aos recursos internos ou qualidades pessoais, mas envolve a interação de aspectos individuais e contextuais. A segunda dificuldade refere-se exatamente à avaliação dos critérios para definir riscos, os recursos de proteção, bem como o quão bem uma pessoa se adapta positivamente aos acontecimentos adversos, o que pode comprometer a inteligibilidade e operacionalização da resiliência (Masten, 2015).

As pesquisas de resiliência têm assumido duas principais estratégias analíticas de investigação: estudos focados na variável e estudos focados nas pessoas (Masten, 2015). A primeira abordagem recorre às análises estatísticas multivariadas, a fim de identificar padrões, mensurando as características pessoais, interpessoais e contextuais. Além disso, busca explorar as ligações entre risco, proteção e os padrões adaptativos, e ainda estabelece relações entre as variáveis preditoras, mediadoras, moderadoras e os resultados adaptativos.

Já a segunda abordagem analítica, com predomínio dos estudos qualitativos, tem o indivíduo como o principal objeto de investigação e busca compreender em profundidade os significados que as pessoas atribuem às suas realidades de vida. Além de capturar os padrões relacionais e contextuais, atrelados aos riscos e às formas de superação que envolve as trajetórias de vida das pessoas. Os estudos centrados na pessoa também podem ser quantitativos, principalmente quando, de forma dedutiva, envolvem a classificação de indivíduos em situações de risco e buscam compará-los a partir de diferentes perfis de adaptação. Ambas as abordagens, focadas na variável e na pessoa, apresentam pontos fortes e fragilidades, o que faz com que alguns estudiosos optem por incluir os dois modelos operacionais em uma mesma pesquisa (e.g., Kubicek, McNeeley, Holloway, Weiss, \& Kipke, 2013; Rodríguez \& Calle, 2013).

Como forma de ampliar o conhecimento acerca da avaliação da resiliência, realizou-se uma revisão integrativa da literatura com o objetivo geral de analisar as estratégias metodológicas para investigação da resiliência nos estudos empíricos com as pessoas LGB. Para responder a tal objetivo realizou-se as seguintes indagações: Como a resiliência tem sido investigada metodologicamente nos estudos empíricos com pessoas LGBs? Especificamente, quais modelos analíticos de investigação da resiliência têm sido utilizados? E quais variáveis, instrumentos e métodos de análise dos dados são mais usados para mensurar e compreender o fenômeno da resiliência?

Uma das questões centrais às pesquisas sobre resiliência na população LGB é a avaliação de como esse construto pode ser investigado metodologicamente, ou seja, mensurado (quantitativamente) e compreendido (qualitativamente). Nesse sentido, acredita-se que esta revisão integrativa da literatura pode contribuir para os dois campos de conhecimentos em questão: sobre resiliência e sobre desenvolvimento de populações LGBs. Ao sintetizar de forma rigorosa e discutir as estratégias metodológicas dos modelos de resiliência e as minorias sexuais, pode-se colaborar para o alinhamento teórico-metodológico desse campo de investigação e fortalecer cada vez mais a inteligibilidade desse construto. Além disso, o constructo da resiliência pode ser um rico modelo conceitual para avaliar a saúde e indicar estratégias de intervenção desenvolvimental na vida da população LGB (Colpitts \& Gahagan, 2016). 


\section{Método}

Trata-se de uma revisão integrativa de literatura e para nortear e sistematizar a execução da revisão seguiu-se as diretrizes enunciadas no protocolo PRISMA (Preferred Reporting Items for Systematic Reviews and Meta-Analysis; Liberati et al., 2009).

Base de Dados e Procedimentos de Coleta de Dados: Elegeu-se as bases de dados PUBMED, PsycINFO, Scientific Electronic Library Online (SciELO), Periódicos Eletrônicos de Psicologia (PePSIC), Literatura Latino-Americana e do Caribe em Ciências da Saúde (LILACS) e IndexPsi, de modo a contemplar tanto a literatura nacional como internacional, incluir tanto estudos da área da saúde como da psicologia, e desse modo, apontar as principais tendências e resultados do tema investigado. A coleta bibliográfica foi realizada primeiramente em agosto de 2015 e posteriormente, em dezembro do mesmo ano, com a inclusão de novos artigos. Os descritores de busca em português com os operadores booleanos e processos de trucagem foram: (resilien\$) AND (homossex\$ OR lesbi\$ OR gay\$ OR bissex\$ OR LGBT OR mesmo sexo) e os respectivos termos em inglês (resilien*) AND (homosex* OR lesb* OR gay* OR bisex* OR LGBT OR same-sex). Mesmo que o estudo não tivesse como objetivo investigar as estratégias metodológicas da resiliência com a população trans (transgênro, transsexual), incluiu-se o termo LGBT por ser uma expressão amplamente utilizada no meio acadêmico e dessa forma, poderia contemplar um número maior de estudos com as minorias sexuais e de gênero.

Seleção dos Estudos: Para serem incluídos os estudos teriam que: (1) ser empíricos - quantitativos, qualitativos ou mistos; (2) ter como objetivo investigar os processos de resiliência na população LGB. Não houve restrição ao período de publicação, dado o estágio inicial das pesquisas de resiliência e as minorias sexuais. Foram excluídos os artigos duplicados; artigos teóricos, capítulos de livro, revisões sistemáticas, narrativas e integrativas da literatura, notícias, documentos técnicos, editoriais, comen- tários, dissertações e teses. Excluiu-se ainda os estudos que, mesmo explorando alguns conceitos relacionados à resiliência (risco, proteção, adaptação positiva), não tinham como objetivo estudar a resiliência propriamente dita. A partir de então, foram examinados os títulos e resumos (abstracts), de acordo com os critérios de inclusão e exclusão estabelecidos. Após essa primeira seleção, todos os artigos foram recuperados na versão completa e, nesse momento, submetidos à avaliação de dois juízes independentes - duas estudantes da pós-graduação (ambas integrantes do grupo de pesquisa do qual as autoras desse artigo fazem parte), seguindo o protocolo intitulado como Teste de Relevância (Azevedo, 2010). Este teste é constituído por quatro itens referentes à qualidade do artigo, considerando os seguintes critérios: clareza do problema de pesquisa; relação do objetivo do estudo com a questão que está sendo investigada; clareza da metodologia e alcance dos objetivos; compatibilidade entre os resultados e metodologia. Incluiu-se mais um item no protocolo considerando se o estudo tinha como objetivo estudar a resiliência e a população LGB. As exclusões foram esclarecidas pelos juízes e as divergências resolvidas em consenso entre eles. Uma vez selecionados os artigos que compuseram o corpus de análise da presente revisão, os mesmos foram analisados e os seus dados foram armazenados em planilha do Excel. A partir daí, iniciou-se o processo de classificação em categorias levando em consideração o objetivo geral do estudo.

Análise e Síntese dos Dados: Para análise dos dados e uma maior compreensão do fenômeno investigado, os estudos foram categorizados seguindo as abordagens analíticas da resiliência propostas por Masten (2015): (1) Estudos centrados na variável; (2) Estudos centrados na pessoa e; (3) Estudos centrados na variável e na pessoa simultaneamente. Como o objetivo principal deste estudo é acerca das estratégias metodológicas para a operacionalização da resiliência, em cada uma das categorias de análise foi observado e discutido o delineamento, as principais variáveis (quando quantitativos), os principais instrumentos (quando qualitativos) e o método de 
análise dos dados utilizado para a investigação da resiliência.

\section{Resultados e Discussão}

A pesquisa inicial nas bases eletrônicas localizou 172 estudos, os quais foram identificados apenas nas bases internacionais: PUBMED $(n=$ $127)$ e PsycINFO $(n=45)$. Após a leitura dos títulos e resumos aplicou-se os critérios de inclusão e exclusão, chegando aos seguintes dados: comentários e relatórios técnicos $(n=9)$; estudos que não problematizavam o tema "resiliência e a população LGB" $(n=38)$, estudos teóricos e de revisão $(n=18)$. Posteriormente a esta primeira seleção, obteve-se um número de 107 estudos, os quais tiveram todos os textos completos recuperados. Estes artigos foram encaminhados aos juízes e submetidos ao protocolo intitulado Teste de relevância (Azevedo, 2010). A partir desta análise houve uma taxa de discordância, de aproximadamente, $6 \%(n=2)$ entre os juízes, sendo esclarecida e resolvida em consenso entre eles. A discordância houve principalmente na compreensão se o artigo tinha ou não a resiliência como foco. Foram excluídos, portanto, 76 artigos. Dentre os principais motivos de exclusão, após o exame dos artigos, destacam-se: estudos que não atendiam ao objetivo da revisão, ou seja, não tinham como objetivo investigar a resiliência no contexto das minorias sexuais (gays, lésbicas e bissexuais); artigos que avaliavam apenas alguns dos indicadores da resiliência (bem-estar, qualidade de vida, fatores de risco, fatores de proteção etc.), mas não a resiliência propriamente dita; estudos que analisavam a resiliência exclusivamente no contexto das minorias de gênero (transgêneros). Finalmente, foram incluídos 31 estudos que compuseram o corpo de análise deste trabalho, sendo: PUBMED $(n=$ $22)$ e PsycINFO $(n=9)$. Não foram localizados artigos de resiliência e as minorias sexuais nas demais bases de dados, o que indica uma escassez de estudos relativos a esse tema no cenário nacional e na América Latina. As referências completas dos estudos incluídos nesta revisão integrativa, estão destacadas com asterisco na seção das Referências.

\section{Estudos Focados na Variável}

Nos estudos focados na variável foi possível identificar principalmente as relações entre as variáveis de risco, de proteção e de adaptação/ajustamento psicossocial específicas à população LGB, bem como exploraram padrões de variância entre características individuais (experiências, interrelações e contexto) que explicam diferentes resultados desenvolvimentais. A Tabela 1 apresenta uma descrição do delineamento, das variáveis utilizadas em cada estudo, divididos pelas variáveis independentes, moderadoras, mediadoras e as variáveis dependentes (outcomes), bem como os métodos de análise dos dados (modelos de correlação, de regressão, mediação e de moderação).

Foram localizados 19 artigos, publicados entre o período de 2003 e 2015, dos quais 9 foram publicados em 2015. Prevaleceram os estudos com delineamento transversal $(n=16)$, embora 3 sejam longitudinais.

\section{Variáveis para Avaliação da Resiliência}

Vários estudos aqui analisados assumiram a resiliência como processo e para mensurá-la utilizaram instrumentos de medidas quantitativas multidimensionais associadas à interação entre os fatores de risco e de proteção (variáveis independentes) e os indicadores de adaptação positiva (variáveis dependentes). A variável de risco frequentemente utilizada nos estudos desta revisão e que está significativamente associada aos resultados desenvolvimentais das minorias sexuais refere-se à homofobia, seja externa (vitimização homofóbica, heterossexismo) e/ou interna (homofobia internalizada; e.g., Herrick et al., 2013; Mereish \& Poteat, 2015). Observou-se ainda que aspectos como rejeição familiar, especialmente entre os jovens e idosos LGB (e.g., Zimmerman et al., 2015), consciência do estigma (Figueroa \& Zorcolla, 2015), ocultação da orientação sexual (Livingston et al., 2015), racismo (Peterson et al., 2014) e falta de recursos socioeconômicos (Fredriksen-Goldsen et al., 2015), também se mostraram associados ao mau ajustamento psicossocial. Ver variáveis utilizadas em cada estudo na Tabela 1. 
Tabela 1

Caracterização dos Estudos Quantitativos de Resiliência e a População LGB por Ano de Publicação

\begin{tabular}{|c|c|c|c|c|c|}
\hline \multirow[t]{2}{*}{ Estudos } & \multirow[t]{2}{*}{ Delineamento } & \multicolumn{3}{|c|}{ Variáveis para avaliação da Resiliência } & \multirow{2}{*}{$\begin{array}{l}\text { Modelos } \\
\text { de análise } \\
\text { dos dados }\end{array}$} \\
\hline & & $\begin{array}{c}\text { Variáveis } \\
\text { Independentes }\end{array}$ & $\begin{array}{c}\text { Variáveis } \\
\text { Dependentes }\end{array}$ & $\begin{array}{c}\text { Variáveis } \\
\text { Mediadoras/ } \\
\text { Moderadoras }\end{array}$ & \\
\hline $\begin{array}{l}\text { Bruce, Harper, \& } \\
\text { Bauermeister (2015) }\end{array}$ & Transversal & $\begin{array}{l}\text { Experiência de estigma; } \\
\text { Estresse pela ocultação } \\
\text { da orientação sexual; } \\
\text { Suporte Social }\end{array}$ & Depressão & $\begin{array}{l}\text { Homofobia } \\
\text { Internalizada; } \\
\text { Desenvolvimento } \\
\text { de identidade } \\
\text { positiva } \\
\text { (indicadora de } \\
\text { resiliência) }\end{array}$ & Mediação \\
\hline
\end{tabular}

Buttram, Surratt, \& Transversal Kurtz (2014)

Figueroa \& Zoccola Transversal (2015)

Fredriksen-Goldsen, Transversal Vitimização e discriminação Kim, Shiu, Goldsen, \& Emlet (2015)

Kosciw, Palmer, \& Transversal Kull (2015)

Mason, Lewis, Winstead, \& Derlega (2015)
Falta de moradia; Severo estresse mental; Abuso de substância; Vitimização; Risco de HIV; Educação; Cobertura de saúde; Acesso à transporte

Assédio, discriminação e rejeição heterossexista; Consciência do estigma ao longo da vida;

Recursos de gerenciamento de identidade (senso positivo de Identidade, revelação de identidade, tempo de divulgação);

Recursos sociais (parceiros ou casados, tamanho da rede social, atividades religiosas ou espirituais, apoio social, conectividade);

Comportamentos de promoção da saúde; (atividade física, atividade de lazer, check-up de rotina, não uso de substância); Recursos socioeconômicos (renda, emprego, e educação).

Abertura da orientação sexual; Vitimização

Assédio, rejeição e discriminação heterosexista; Homofobia Internalizada Restrições Sociais
Personal

mastery

(Resiliência)

Saúde física; saúde mental

Robustez

Moderação

(depressão, ansiedade e somatização) (medida de resiliência)

Saúde física e mental

Resultados

Autoestima; Mediação acadêmicos (frequência e notas escolares)

\section{Autoestima coletiva}

\section{Depressão}

Regressão 


\begin{tabular}{|c|c|c|}
\hline $\begin{array}{l}\text { Shilo, Antebi } \\
\& \text { Mor (2015) }\end{array}$ & Transversal & $\begin{array}{l}\text { Homofobia Internalizada } \\
\text { Revelação da orientação } \\
\text { sexual; Assédio gays; } \\
\text { Suporte familiar e dos } \\
\text { amigos; Conexão com a } \\
\text { comunidade LGBTQ }\end{array}$ \\
\hline
\end{tabular}

Zimmerman, Longitudinal

Darnell, Rhew, Lee,

\& Kaysen (2015)

Peterson et al.

(2014)

Reisner, Biello, Perry, Gamarel, \& Mimiaga (2014)

Herrick et al.

(2013)

Walker \&

Longmire-Avital

(2013)

Fredriksen-Goldsen et al. (2012)

Kurtz, Buttram, Surratt, \& Stall (2012)

Poteat, Mereish, DiGiovanni, \& Koenig (2011)
Identidade LGB; Exteriorização da orientação coletiva; Conexão sexual

Homofobia; Racismos; Experiência de vida Dados Sociodemográficos

Transversal

Risco: Uso de álcool; Bullying; Depressão; Proteção: Apoio familiar; Apoio escolar; Engajamento comunitário; Equipe esportiva; Identidade LGBTQ; Variáveis sociodemográficas

Longitudinal

Violência pelo parceiro íntimo; Homofobia internalizada; Dados sociodemográficos

Transversal Fé religiosa; Saúde mental (Depressão e Ansiedade)

Transversal Vitimização; Estigma internalizado; Ocultação da Orientação sexual; Suporte social; Tamanho da rede social; Resultados de saúde (exame de rotina, barreiras financeiras aos cuidados de saúde, obesidade, fumar, beber em excesso, atividades físicas).

Transversal Estresse mental (Ansiedade e Depressão); Escape cognitivos (uso de álcool e/ ou drogas juntamente com $o$ sexo); Vitimização

Transversal Vitimização geral; Vitimização homofóbica; Suporte parental Dados sociodemográfico com a comunidade LGBTQ

Saúde mental

Regressão

Rejeição Moderação

Familiar

Resiliência

Infecção HIV

Correlação

e regressão

Autoagressão

e tentativa de suicídio

Regressão

Depressão; Uso de substância;

Comportamento sexual compulsivo

Resiliência

Homofobia Moderação Internalizada

Depressão;

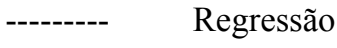

Deficiência/

Incapacidade;

Pobre saúde geral.

Resiliência

Regressão

(Comportamentos de coping; autoeficácia e engajamento social)

Preocupações educacionais (notas médias, ausência escolar, importância da graduação)

\footnotetext{
Variáveis Mediação mediadoras (suicídio; pertencimento escolar) Variável moderadora (suporte parental; vitimização)
} 
Gwadz et al. (2006)

Russell \& Richards (2003)
Transversal

Vitimização na infância;

Eventos de vida

Características sociodemográficas
Transversal

$$
\begin{aligned}
& \text { Estressores associados } \\
& \text { à campanhas anti-gays } \\
& \text { (homofobia, homofobia } \\
& \text { internalizada, divisão } \\
& \text { comunitária, senso de } \\
& \text { perigo); Resiliência } \\
& \text { associado à campanhas } \\
& \text { anti-gays (enfrentamento } \\
& \text { da homofobia, expressão } \\
& \text { de afeto, testemunhos de } \\
& \text { sucesso, comunidade LGB) }
\end{aligned}
$$

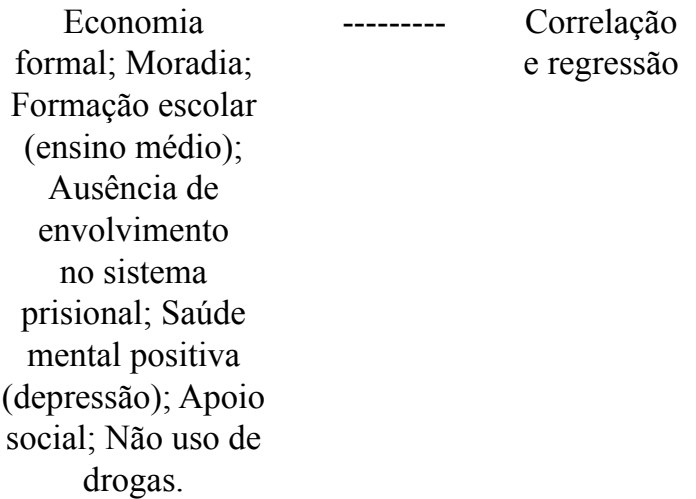

Correlação
As principais variáveis relacionadas aos $f a-$ tores de proteção e que têm o papel de favorecer os processos de resiliência entre as pessoas LGB, referem-se aos recursos protetivos internos (pessoais) e externos (contextuais). Sobre os recursos de proteção internos identificou-se, por exemplo: a abertura emocional, a esperança, o otimismo (Kwon, 2013); senso positivo em relação à orientação sexual (FredriksenGoldsen et al., 2015; Kosciw et al., 2015); fé/ espritualidade (Walker \& Longmire-Avital, 2013), dentre outros (ver Tabela 1). Por sua vez, os fatores de proteção externos continuam a ser incorporados de forma tímida nas investigações. Localizou-se, por exemplo, o ajustamento diádico (Bonanno, Moskowitz, Papa, \& Folkman, 2005); o apoio/aceitação familiar (Reisner et al., 2014); percepção do suporte social (e.g., Mustanski et al., 2011).

No que se refere às variáveis para avaliar a adaptação positiva (ver Tabela 1), os estudos utilizaram tanto indicadores de adaptação interna (e.g., saúde mental/psicológica, bem-estar subjetivo) como indicadores externos, relacionados ao cumprimento de tarefas desenvolvimentais esperadas socialmente e habilidades relacionais (e.g., conexão comunitária, sucesso escolar/acadêmico, conduta social, comportamento sexual de risco, qualidades acadêmicas e econômicas). A presença de saúde mental (e.g., depressão, ansiedade), todavia, foi destacada como os indicadores mais frequentemente utilizados para avaliar a adaptação positiva desta população.

Ressalta-se ainda que alguns estudos analisados nesta revisão mesmo que conceitualmente reconheçam a resiliência como processo, assumiram que ela pode ser medida diretamente, em uma única dimensão, sendo explicada como o próprio resultado adaptativo (e.g., Buttram et al., 2014; Kurtz et al., 2012; Walker \& Longmire-Avital, 2013), ou mesmo como efeito "tampão" saliente que pode minimizar os efeitos dos riscos e levar a um melhor estado de saúde (Figueroa \& Zocolla, 2015; Peterson et al., 2014). Por exemplo, dois desses estudos utilizaram a Resilience Scale, desenvolvida por Wagnild e Young (1993), para avaliar o nível de adaptação psicossocial positiva nos acontecimentos significativos da vida (Peterson et al., 2014; Walker \& Longmire-Avital, 2013).

Com base no exposto percebe-se que, nestes estudos que mensuraram diretamente a resi- 
liência, ela foi tomada como resultado/desfecho. Assim, não é a adaptação positiva, através dos indicadores de saúde, de bem-estar ou mesmo de cumprimento de tarefas de desenvolvimento que sugere a resiliência. Mas esta que indica a adaptação positiva, a partir de suas capacidades pessoais. Assume-se, todavia, que a resiliência é um processo que leva à adaptação positiva. Ela não é o ponto final ideal que os indivíduos precisam chegar, e portanto, não é mantida em um estado permanente. Em vez disso, ela é um processo contínuo e que envolve os processos interacionais entre os indivíduos e seu meio ambiente e que pode ser desenvolvida ao longo do tempo, sendo a adaptação positiva o seu desfecho (Hill \& Gunderson, 2015).

Apesar dos notáveis avanços nos estudos de resiliência com foco na variável e as minorias sexuais, incorporando uma perspectiva mais positiva e dando destaque às potencialidades dessa população, muitos estudos continuam a operacionalizá-la metodologicamente considerando apenas variáveis com atributos individuais como promotores do desenvolvimento psicossocial. Além disso, a própria adaptação, no geral, também foi avaliada com base nos critérios mais individuais, relacionados à saúde mental. Ressalta-se, porém, que a noção de resiliência associada às características individuais pode minimizar a importância do ambiente social e responsabilizar unicamente indivíduo pelo seu (in) sucesso, desconsiderando o papel das forças sociais, políticas, econômicas e culturais que podem promover ou mesmo inibir os processos de resiliência. Certamente, dentre tantos outros fatores, a inclusão de políticas públicas eficientes são agentes facilitadoras da resiliência, sobretudo entre os grupos socialmente marginalizados (Ungar, 2015).

\section{Modelos de Análise dos Dados}

Três principais modelos operacionais foram identificados para analisar os processos de resiliência: (a) modelo compensatório ou de efeito principal $(n=12)$; (b) modelo mediador $(n=3)$ e; (c) modelo moderador $(n=4)$. Dos estudos empíricos investigados, 12 adotaram o modelo compensatório ou de efeitos principais para ex- plicar a resiliência, sobretudo a partir da análise do efeito direto das variáveis independentes (risco e/ou proteção) sobre as variáveis dependentes (adaptação/ajustamento positivo). Para chegar a tais explicações - não causais - os métodos de correlação e regressão simples foram as principais estratégias analíticas utilizadas (Ver Tabela 1). Utilizando o modelo de correlação, por exemplo, Reisner et al. (2014) observaram que o comportamento de autoflagelo foi associado positivamente ao suicídio em adultos LGBTQ (lésbicas, gays, bissexuais, transgêneros e queer) e heterossexuais. Por sua vez, a análise de regressão mostrou que o apoio familiar foi associado a uma maior probabilidade de comportamentos de autoflagelos e a tendências suicidas. Dessa forma, um fator de proteção (apoio familiar) agiu no sentido contrário ao fator de risco (autoflagelo), compensando os efeitos negativos nos resultados de ajustamento psicossocial (suicídio).

Os estudos que adotaram o modelo de mediação $(n=3)$, refletiram a sequência em que as variáveis indepententes (fatores de risco e de proteção) causam efeitos indiretos em uma ou mais variáveis mediadoras, e este efeito, propaga-se às variáveis de desfecho (adaptação positiva). Por exemplo, a revelação de identidade LGBT de jovens alunos para colegas e professores na escola foi relacionada à altas taxas de vitimização, mas também associada à autoestima elevada, o que colaborou para a taxa de depressão mais baixa (Kosciw et al., 2015). Desse modo, as variáveis mediadoras (vitimização e autoestima) foram influenciadas pela variável independente (revelação da orientação sexual) e indiretamente transmitiram um efeito sobre a adaptação (depressão). Em outro estudo de mediação observou-se que a homofobia internalizada mediou parcialmente o efeito das experiências de estigma relacionados à orientação sexual em relação aos sintomas depressivos (Bruce et al., 2015). Além disso, os resultados sugerem que pessoas LGBT que vivenciavam experiências de estigma e em decorrência disso, vivenciavam o estresse pela ocultação da orientação sexual, acabavam por buscar apoio social de outras pessoas LGBT, o que colaborava para o desenvolvimento da identidade positiva. Esses resultados apoiam a 
ideia de que os efeitos indiretos da vitimização homofóbica sobre as pessoas LGB são mediados pela homofobia internalizada, trazendo mais danos à saúde psicológica da população LGB.

Já os estudos de moderação $(n=4)$ evidenciaram como uma variável moderadora afeta a direção e/ou a força da relação entre variáveis independentes (risco e proteção) e a variável dependente/desfecho (adaptação positiva). Em um estudo de regressão longitudinal com 843 mulheres adultas jovens lésbicas e bissexuais, por exemplo, observou-se que ao revelarem a orientação sexual para a família, mesmo com alto índice de rejeição familiar (variável moderadora), demonstraram resiliência ao encontrarem conexões com a comunidade de minorias sexuais e vivenciarem autoestima coletiva (Zimmerman et al., 2015). Todavia, quando o estigma preocupa, aumentam as motivações para ocultar a orientação LGBT, bem como crescem outros fatores de risco de identidade sexual, mostrando menos conexão com a comunidade. Outro exemplo pode ser observado no estudo de Mereish e Poteat (2015) em que jovens LGBT que estabeleciam relações de crescimento com amigos próximos (LGBT ou heterossexual) estariam associadas com menor sofrimento psicológico para as minorias sexuais com baixo nível de homofobia internalizada (variável moderadora). Para os participantes com níveis elevados de homofobia, as relações de crescimento com um amigo LGBT foi associada com menos sofrimento psíquico, mas não com um amigo heterossexual.

Embora a identificação prévia da associação das variáveis de risco e de proteção como preditoras dos resultados adaptativos da população LGB seja importante, é preciso reconhecer que o impacto desses fatores pode ser diferente entre os indivíduos LGB, suas famílias e comunidades. Em relação aos riscos, por exemplo, apesar deles significarem uma ameaça à qualidade da adaptação nos processos de resiliência, ressalta-se que o risco é um termo probabilístico, ou seja, na sua presença aumenta-se a probabilidade de um resultado negativo para membros de um determinado grupo, mas não necessariamente indicam a natureza exata da ameaça para um in- divíduo (Wright \& Masten, 2015). Os riscos são aspectos multifacetados e uma avaliação mais rigorosa de seus efeitos precisam considerar o seu efeito cumulativo, a cronicidade, a complexidade ecológica que a pessoa está envolvida diante da situação de ameaça, bem como a relevância dos fatores culturais e contextuais que afetam as pessoas diante da experiência de risco (Ungar, 2015; Wright \& Masten, 2015).

Além disso, é importante ressaltar que, com base nos resultados analisados nesta revisão, uma mesma variável pode funcionar como um risco/vulnerabilidade num dado contexto, mas em outras circunstâncias, como um fator de proteção. Por exemplo, a revelação de identidade LGBT pode estar relacionada à altas taxas de vitimização, mas também pode estar associada à autoestima elevada (e.g., Kosciw et al., 2015). Ou seja, revelar a orientação sexual pode ser um fator de risco ao colocar as minorias sexuais diante do preconceito homofóbico. Por outro lado, esse processo de afirmação identitária pode aumentar a autoestima e colaborar para melhores resultados adaptativos.

A investigação metodológica da resiliência exige, portanto, uma complexa avaliação dos riscos, dos aspectos protetivos e da adaptação positiva. A inconsistência na operacionalização desses fatores pode prejudicar a compreensão e a generalização dos resultados empíricos (Masten, 2015). Os estudos de mediação e moderação revelam a sutileza e complexidade na avaliação do conjunto de sistemas e processos que colaboram ou não para os processos de resiliência em minorias sexuais. Os seus resultados sinalizam que não é possível estabelecer uma relação de causalidade linear e de aparente determinismo entre os fatores de risco, proteção de ajustamento biopsicossocial entre as pessoas LGB, mas envolve a compreensão de múltiplos percursos e contextos de riscos e de superação.

\section{Estudos Focados na Pessoa}

Aqui, a resiliência pode ser operacionalizada mediante os significados que a população LGBT atribui aos desafios que vivenciam nas suas trajetórias de vidas, bem como as estratégias que utilizam para superar os eventos desafiadores 
(e.g., Sung, Szymanski, \& Henrichs-Beck, 2015). O objetivo, portanto, não é generalizar resultados, mas identificar a variedade dos contextos de adversidades vividas, bem como as histórias de enfrentamento e superação de pessoas reais e que apontam para a complexidade dos processos de resiliência que envolvem as pessoas LGB. A Tabela 2 apresenta uma descrição dos instrumentos utilizados em cada estudo, os métodos de análise dos dados e as principais categorias temáticas.

Dentre os artigos selecionados nesta revisão, oito foram centrados na pessoa, todos qualitativos, com delineamento transversal e publicados entre os anos de 2003 e 2015.

\section{Instrumentos para Coleta de Dados}

Em relação aos instrumentos utilizados nos estudos qualitativos, identificou-se principalmente a entrevista - semiestruturada e aberta. Privilegiou-se, dessa forma, as histórias de vida das pessoas LGB, fornecendo informações sobre os desafios significativos vividos ao longo da trajetória de vida, mas sobretudo, apontou-se para os recursos e sistemas de proteção acessados ao longo do tempo e que refletem nos processos de resiliência. Exemplos de questões que os autores utilizaram para indagar sobre esses processos foram: "Quais foram os processos e fatores-chave na integração da sua identidade de ser tanto gay/ lésbica e cristão?” (Foster et al., 2015); bem como "Por favor, diga-me alguns desafios que você vivencia no dia-a-dia como lésbica/bissexual América-Asiática"; e "Por favor, conte-me sobre as maneiras de lidar e/ou resistir aos desafios do dia-a-dia que você enfrenta como uma lésbica/bissexual Americana Asiática?" (Sung et al., 2015). Ou seja, mais do que uma entrevista, os pesquisadores propuseram que os participantes relatassem as suas histórias de vida, de modo a captar em maior profundidade a realidade das minorias sexuais.

\section{Métodos de Análise dos Dados e Categoria Temáticas}

Os principais métodos de análise nos estudos focados na pessoa nesta revisão, referem-se sobretudo à análise de conteúdo $(n=4)$, grounded-theory $(n=3)$ e análise fenomenológica $(n$ $=1$ ). Essas estratégias metodológicas privilegiaram a riqueza dos significados atribuídos à experiência de vida, considerando o caráter dinâmico, processual e provisório da resiliência (Yunes, 2003). Desse modo, de forma indutiva, as categorias de análise emergiram a partir das histórias de vida narradas dos participantes, e não a partir de uma teoria prévia. A Tabela 2 apresenta as categorias temáticas de cada estudo.

Acerca das categorias temáticas, observouse que elas estavam relacionadas principalmente à descrição dos desafios vividos, bem como aos recursos protetivos que acabam por colaborar para os processos adaptativos das minorias sexuais. À semelhança dos estudos focados na variável, o principal fator de risco relatado pelos participantes relacionou-se às experiências de discriminação homofóbica, seja no contexto familiar, escolar, institucional e cultural (e.g., DiFulvio, 2011; Erhard \& Ben-Ami, 2015). Os participantes descreveram por exemplo, terem sofrido bullying homofóbico, serem alvos de violência por terem aparência e agir diferente da norma estabelecida socialmente ou mesmo estar acompanhado de alguém LGBT, o que acaba por gerar uma desconexão dessas pessoas com as suas comunidades (e.g., DiFulvio, 2011). Além disso, o gerenciamento das múltiplas identidades de minorias(LGB, imigrante, negro) revelam tensões cotidianas e fontes de estresses nas suas vidas, especialmente em função do heterossexismo, racismo e sexismo (e.g., Bowleg et al., 2003). Ver outras categorias temáticas na Tabela 2.

Os estudos aqui analisados também exploraram as diversas estratégias que as pessoas LGB utilizaram para enfrentar e superar os desafios e estresse que acompanhavam as suas vidas e as colocavam em uma situação de risco, por exemplo: gerenciar identidades de minorias e buscar empoderamento (Sung et al., 2015); encontrar conexão com a comunidade LGBT e com a comunidade racial/étnica (Gray et al., 2015); ter relações de apoio social; sentimentos de singularidade, autoestima, comportamentos e competências sociais, felicidade, otimismo e humor (Bowleg et al., 2003); engajamento em pro- 
Tabela 2

Caracterização dos Estudos Qualitativos (focados na pessoa) de Resiliência e a População LGB por Ano de Publicação

\begin{tabular}{|c|c|c|c|c|}
\hline Estudos & Delineamento & Instrumentos & Categorias temáticas & $\begin{array}{c}\text { Modelos de } \\
\text { análise dos dados }\end{array}$ \\
\hline $\begin{array}{l}\text { Farr, Crain, } \\
\text { Oakley, Cashen, } \\
\text { \& Garber } \\
(2016)\end{array}$ & Transversal & $\begin{array}{l}\text { Entrevistas } \\
\text { semiestruturadas } \\
\text { e sessões de } \\
\text { videotapes }\end{array}$ & $\begin{array}{c}\text { Categorias de análise: (a) Os sentimentos } \\
\text { de diferença, (b) Microagressões, e (c) } \\
\text { Resiliência (conceituações positiva acerca } \\
\text { da família) }\end{array}$ & Análise temática \\
\hline $\begin{array}{l}\text { Erhard \& } \\
\text { Ben-Ami, } \\
(2015)\end{array}$ & Transversal & $\begin{array}{c}\text { Entrevista } \\
\text { semiestruturada }\end{array}$ & $\begin{array}{l}\text { Fatores de proteção ecológica (coping grupo } \\
\text { minoritário, comunidade virtual defesa } \\
\text { LGB, e aceitação dos pares heterossexuais } \\
\text { na escola); Mecanismos de enfrentamento } \\
\text { (avaliação cognitiva de incidentes anti-LGB } \\
\text { na escola, comunicação assertiva, tornando-se } \\
\text { um advogado em nome da comunidade LGB; } \\
\text { ignorar taticamente; questionando e resistindo } \\
\text { aos rótulos sexuais) }\end{array}$ & $\begin{array}{c}\text { Teoria } \\
\text { fundamentada }\end{array}$ \\
\hline $\begin{array}{l}\text { Foster, } \\
\text { Bowland, \& } \\
\text { Vosler (2015) }\end{array}$ & Transversal & $\begin{array}{l}\text { Entrevista } \\
\text { aberta }\end{array}$ & $\begin{array}{l}\text { Transformando significados teológicos; } \\
\text { Descoberta de uma comunidade segura; } \\
\text { Permanecer em comunidade; Buscando } \\
\text { uma nova comunidade; Trabalhando } \\
\text { para a justiça social }\end{array}$ & $\begin{array}{c}\text { Teoria } \\
\text { fundamentada }\end{array}$ \\
\hline $\begin{array}{l}\text { Gray, } \\
\text { Mendelsonhn, } \\
\text { \& Omoto } \\
(2015)\end{array}$ & Transversal & $\begin{array}{c}\text { Entrevista } \\
\text { semiestruturada }\end{array}$ & $\begin{array}{c}\text { (Des) conexão com a comunidade LGBT; } \\
\text { (Des) conexão com a comunidade latina; } \\
\text { Desafios e estratégias interseccionais; } \\
\text { Bem-estar, força e resiliência }\end{array}$ & $\begin{array}{l}\text { Análise de } \\
\text { conteúdo }\end{array}$ \\
\hline $\begin{array}{l}\text { Sung et al. } \\
(2015)\end{array}$ & Transversal & $\begin{array}{l}\text { Entrevista } \\
\text { aberta }\end{array}$ & $\begin{array}{l}\text { Desafios (Viver com múltiplas identidades } \\
\text { minoritárias; Invisibilidade; Estereótipos } \\
\text { sexuais, Fantasias e Fetichização; Enfrentando } \\
\text { a opressão baseada em orientação sexual); } \\
\text { Estratégias de enfrentamento (Estratégia } \\
\text { de gestão de identidade; Estratégias } \\
\text { de empoderamento); Aspectos positivos } \\
\text { (fontes de força sociocultural; Insight } \\
\text { e empatia consigo e com os outros) }\end{array}$ & $\begin{array}{l}\text { Análise de } \\
\text { conteúdo }\end{array}$ \\
\hline $\begin{array}{l}\text { Harper, Bruce, } \\
\text { Hosek, } \\
\text { Fernandez, \& } \\
\text { Rood }(2014)\end{array}$ & Transversal & $\begin{array}{c}\text { Entrevista } \\
\text { semiestruturada }\end{array}$ & $\begin{array}{l}\text { Engajamento em processos que promovem } \\
\text { saúde cognitiva; Manter práticas } \\
\text { comportamentais saudáveis; (Receber } \\
\text { apoio social de outros; Empoderar outros } \\
\text { homens jovens gays / bissexuais }\end{array}$ & $\begin{array}{c}\text { Análise } \\
\text { Fenomenológica }\end{array}$ \\
\hline DiFulvio (2011) & Transversal & $\begin{array}{l}\text { Entrevistas e } \\
\text { grupos focais }\end{array}$ & $\begin{array}{l}\text { Conexão social a um indivíduo (auto } \\
\text { afirmação); Conexão social a um grupo } \\
\text { (encontrar outras pessoas como você); } \\
\text { Conexão social a um grupo (trabalhar } \\
\text { para a mudança) }\end{array}$ & $\begin{array}{l}\text { Análise de } \\
\text { conteúdo }\end{array}$ \\
\hline $\begin{array}{l}\text { Bowleg, Huang, } \\
\text { Brooks, Black, } \\
\text { \& Burkholder } \\
(2003)\end{array}$ & Transversal & $\begin{array}{c}\text { Entrevista } \\
\text { semiestruturada }\end{array}$ & $\begin{array}{l}\text { Múltiplo estresse de minoria (racismo, } \\
\text { sexismo, heterossexismo); Resiliência } \\
\text { (contexto ambiental externo; processos } \\
\text { interacionais pessoa-ambiente; características } \\
\text { internas; processos de resiliência; resultado de } \\
\text { vida positiva; relações socialmente de apoio) }\end{array}$ & $\begin{array}{c}\text { Teoria } \\
\text { fundamentada }\end{array}$ \\
\hline
\end{tabular}


cessos que promovem saúde cognitiva; manter práticas comportamentais saudáveis; empoderar outros homens jovens gays / bissexuais (Harper et al., 2014); conexão individual e a afiliação a grupos serviram para a afirmação da identidade e forneceram um fórum em que a luta pessoal se transforma em ação coletiva (DiFulvio, 2011).

As categorias temáticas dos estudos qualitativos também revelaram a capacidade de adaptação das pessoas LGB, mostrando saúde e bem-estar, mesmo após lidar com as experiências negativas que acompanham as suas vidas. Por exemplo, em estudo com 13 gays imigrantes latinos explorou-se qualitativamente as fontes de estresse e desafios, bem como as oportunidades relacionados à resiliência desse grupo de minoria (Gray et al., 2015). Em seus relatos afirmaram que os desafios foram vividos como fonte de crescimento e desenvolvimento pessoal, proporcionando uma sensação de força e realização. Isso aconteceu, sobretudo, porque procuraram fontes de apoio social e comunitário, cultivaram as relações sociais, buscaram recursos psicoterapêuticos e trabalharam a autoaceitação e orgulho de si mesmos.

Com base nos dados desta revisão, percebe-se, portanto, que os estudos com foco na pessoa buscaram compreender a complexidade que envolve as dimensões de enfrentamento e superação frente às experiências de dor e sofrimento vivenciadas pelas famílias LGB. Um destaque dessas investigações foi a ênfase aos fatores de proteção, incorporando uma perspectiva salutogênica no desenvolvimento dinâmico da resiliência entre as pessoas LGB, flexibilizando as noções patológicas associados à homossexualidade e iluminando os seus aspectos saudáveis e histórias de sucesso, tanto a nível individual, familiar, como culturalmente. Ademais, o entendimento de como essas pessoas superam as adversidades pode colaborar na descoberta de ações preventivas e intervencionistas, a fim de reforçar os sistemas de proteção e reduzir riscos presentes no cotidiano das minorias sexuais.

Ressalta-se que os estudos qualitativos desta revisão avançam por destacarem aspectos culturais e contextuais específicos da resiliência em pessoas LGB, com atenção à influência da cultura nos fatores de risco (e.g., contexto múltiplo de minorias - sexual, racial, étnico) e nos recursos protetivos (e.g., resgate da identidade cultural).

Os estudos focados na pessoa facilitaram, portanto, a exploração de ricos dados contextuais sobre a vida das minorias sexuais, fornecendo uma imagem holística e aprofundada das vidas das pessoas LGB que viveram/vivem sob adversidades. Enquanto que os estudos centrados na variável são uma fotografia da resiliência, tirada em um dado momento da vida das pessoas LGB, informando a relação entre variáveis de risco, proteção e adaptação positiva, nos estudos qualitativos tem-se um conjunto de fotografias ou um filme contado pelo próprio participante, relatando de forma singular e aprofundada a pluralidade de acontecimentos cotidianos presente nas suas ações individuais, familiares e culturais.

\section{Estudos Focados na Pessoa e na Variável}

Quatro estudos desta revisão integraram os modelos centrados na pessoa e na variável para avaliar metodologicamente a resiliência (Ver Tabela 3). Dois desses estudos buscaram, quantitativamente, encontrar aspectos comuns e diferenciais entre as variáveis de predição (risco e proteção) e as variáveis de desfecho (adaptação); e qualitativamente, de forma aprofundada compreender os processos de resiliência relatados a partir do significado que os participantes atribuem às suas experiências de vida (Kubicek et al., 2013; Rodríguez \& Calle, 2013).

Rodríguez e Calle (2013), por exemplo, realizaram um estudo descritivo e transversal com o objetivo de explorar os aspectos relacionados à saúde mental e à resiliência em 44 jovens gays, lésbicas e bissexuais. $\mathrm{Na}$ pesquisa quantitativa, utilizaram um questionário auto aplicável e exploraram questões como: aspectos da identidade gay; indicadores de opressão internalizada; discriminação; o conhecimento da família sobre a orientação sexual; saúde mental e comportamentos associados com o suicídio. Além disso, aplicaram uma escala para mensurar a resiliência desenvolvida por Lever e Valdez (2010), cons- 


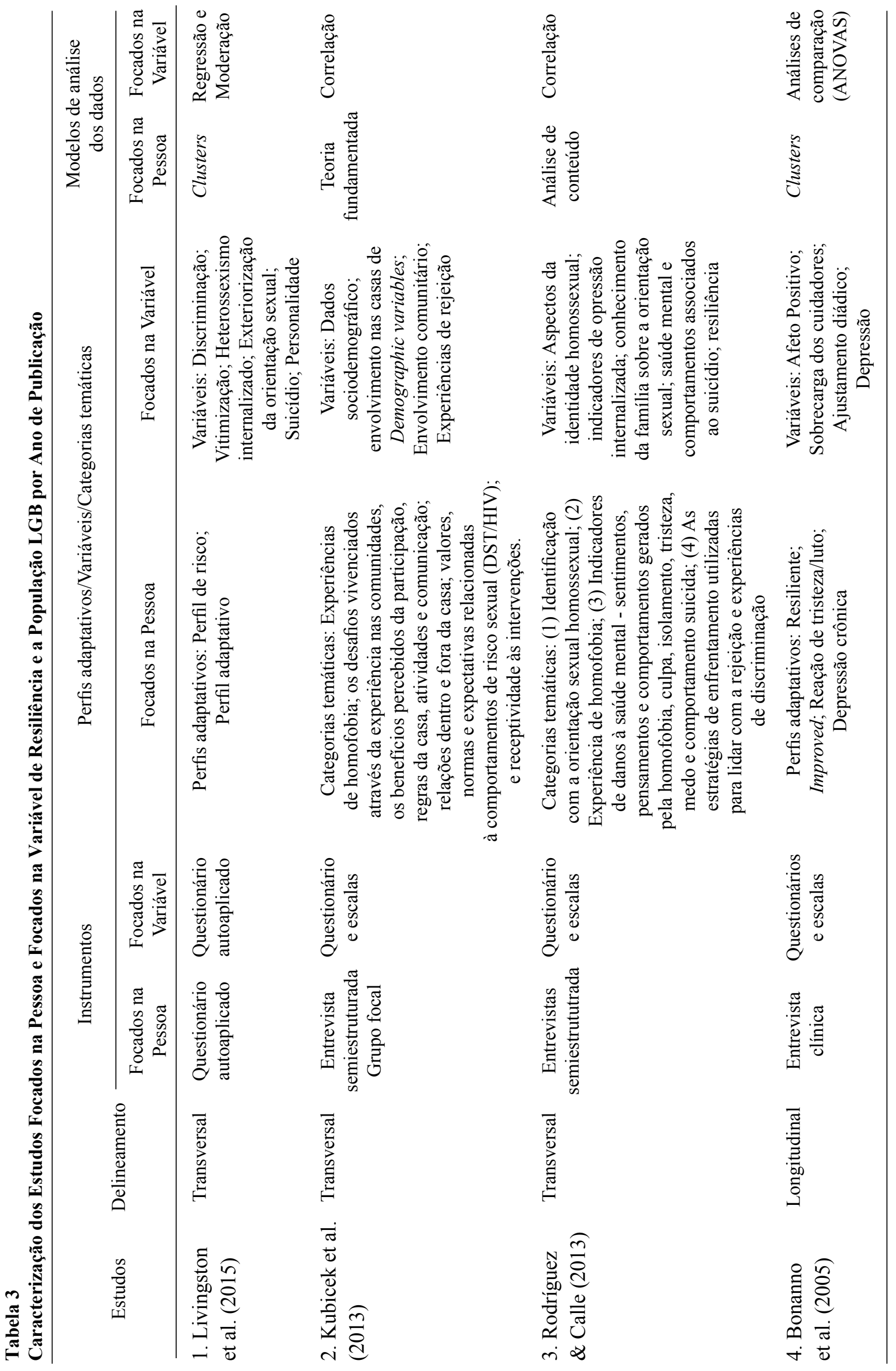


tituída por cinco dimensões: força e confiança, competência social, suporte familiar, suporte e estrutura social. Sob a justificativa de ampliar a reflexão e explorar o significado atribuído às experiências levantadas no estudo quantitativo, os autores realizaram entrevistas semiestruturadas. Os resultados suportam a ideia de que a experiência de ter uma orientação sexual diferente da heteronorma apresenta desafios específicos para estes jovens.

Por fim, outros dois estudos buscaram verificar semelhanças e diferenças nas diferentes trajetórias, identificando perfis de adaptação diante do contexto de risco - luto e vitimização homofóbica, respectivamente (Bonanno et al., 2005; Livingston et al., 2015) e posteriormente, a partir da identificação de grupos adaptativos, estabeleceram relações entre variáveis preditoras e de desfecho. Por exemplo, Livingston et al. (2015), através de uma análise de clusters, traçaram empiricamente perfis distintos de personalidade entre jovens adultos LGB: adaptáveis (neuroticismo inferior e maior extroversão, amabilidade, consciência, e franqueza); e em risco (neuroticismo mais elevado e amabilidade, conscienciosidade e abertura e extroversão inferior). Posteriormente, as regressões logísticas sugeriram que o grupo com personalidade adaptativa apresentava menor risco de tentativa de suicídio diante de situações de vitimização do que o grupo de risco.

Observou-se, dessa forma, que ainda é limitado o número de estudos que combinaram os modelos focados na variável e na pessoa. Para Masten (2015), a junção desses dois processos analíticos é uma grande promessa para os estudos de resiliência, ao explicitar e delinear os múltiplos percursos entre os indivíduos ao longo do tempo, e aumentar a capacidade de avaliar os diversos recursos que refletem os processos resilientes. Quantitativamente, pode-se potencializar os dados estatísticos e buscar aspectos específicos e diferenciais entre preditores e os resultados, além de sugerirem padrões universais na negociação para alcançar resultados desenvolvimentais que são culturalmente relevantes. Qualitativamente, as análises podem captar os padrões marcantes na vida das pessoas e suas comunidades e encontrar uma maior heterogeneidade nos processos de resiliência, ao extrair através das próprias histórias de vida das pessoas as estratégias de enfrentamento e superação das adversidades vividas ao longo do tempo.

\section{Considerações Finais}

Este estudo teve como objetivo analisar as estratégias metodológicas para investigação da resiliência nos estudos empíricos com as pessoas LGB, a partir de uma revisão integrativa da literatura. Dos estudos selecionados foram localizadas pesquisas com foco na variável, estudos com foco na pessoa e, em menor proporção, estudos mistos, centradas na pessoa e na variável simultaneamente.

De forma geral, os resultados dessa revisão integrativa da literatura revelam a complexidade e multiplicidade dos caminhos metodológicos presentes nos estudos de resiliência com as minorias sexuais. A sua medição e/ou compreensão não é uma tarefa simplista, mas passa pela observação e diálogo entre os fatores de risco, de proteção e de adaptação positiva, além de envolver dialeticamente os diferentes níveis e contextos envolvidos: individual, familiar, comunitário e cultural.

Apesar dos avanços metodológicos para investigar a resiliência nos estudos com a população LGB, ao analisar a complexidade envolvida nesse processo e ainda dar destaque às potencialidades das minorias sexuais, percebeu-se alguns limites nos estudos revisados e que merecem ser destacados, a fim de indicar possibilidades para futuras investigações. Primeiramente é importante considerar que os avanços na pesquisa da resiliência, dependem, em certa medida, da existência de uma definição mais consensual a seu respeito. Ou seja, como foi observado ao longo dessa revisão, ainda existem certas divergências no conceito de resiliência, que ora é definida como processo, resultado da interação entre risco e proteção e tendo a adaptação positiva como o resultado dessa interação. Ora, porém, a resiliência é explicada como o próprio resultado de adaptação (e.g., Buttram et al., 2014), ou ainda como um «tampão» saliente 
que pode amortecer os efeitos dos riscos e levar a um melhor estado de saúde das pessoas LGB (Peterson et al., 2014; Rodríguez \& Calle, 2013). Desse modo, essas variações conceituais dificultam a investigação da resiliência e podem comprometer a sua operacionalização, bem como abrem possibilidades para o questionamento da sua aplicabilidade na intervenção e promoção de saúde das minorias sexuais (Colpitts \& Gahagan, 2016).

Os desafios metodológicos, em parte, também se encontram pela qualidade das informações que são recolhidas. Dessa forma, o uso de instrumentos, bem como a adequação na sua aplicabilidade se fazem pertinentes para captar em profundidade as nuances dos processos de resiliência das pessoas LGB. A partir dos dados analisados percebeu-se que as pesquisas com foco nas variáveis continuam a utilizar variáveis e instrumentos de medida com ênfase nos aspectos individuais para captar a resiliência. Todavia, ela não é uma condição de indivíduos sozinhos (Ungar, 2015). Os modelos analíticos precisam reconhecer, portanto, a natureza complexa e multidimensional que acompanha a vida das minorias sexuais e dessa forma, iluminar os recursos que envolvem o seu contexto ecológico (familiar, comunitário, cultural, político, legal) na relação com as suas experiências pessoais. Ao se privilegiar a dimensão mais individual em detrimento do social, as pesquisas continuam a contribuir para a invisibilidade das necessidades das minorias sexuais (Meyer, 2015).

Análises estatísticas mais avançadas também são necessárias a fim de apreender aspectos complexos que envolvem a vida das pessoas LGB. Por exemplo, recomenda-se o uso de modelagem multinível (MLM) nas pesquisas com foco na variável para investigar a interdependência dos dados entre pares familiares, conjugais e parentais; análises de clusters, nos estudos centrados na pessoa para delinear os perfis ou trajetórias de adaptação das minorias sexuais; além do avanço nos estudos de mediação e moderação. Já nos estudos centrados na pessoa, sobretudo qualitativos, observou-se que a entrevista (aberta, semiestruturada e estruturada) foi o principal instrumento para a coleta de dados. Su- gere-se, todavia, o cruzamento de diversos métodos de recolha dos dados com vistas a captar de forma criativa e em profundidade a experiência de vida das pessoas LGB. Por exemplo, o uso de narrativas, fotografias, diário de campo, inserção ecológica e filmes são exemplos de instrumentos que podem permitir que se alcance indicadores qualitativos mais consistentes. $\mathrm{O}$ cruzamento de dados é importante para obter insights sobre a experiência multidimensional das minorias sexuais. Outra limitação é que a maioria dos estudos utilizou um desenho transversal em suas investigações. Dessa forma, é necessário ter cautela em atribuir direções causais às associações entre os fatores de risco, de proteção e adaptação que foram encontradas. Estudos longitudinais de resiliência são cada vez mais indicados nas pesquisas com as pessoas LGB, a fim de apontar se, por exemplo, os recursos de proteção contribuem para o seu desenvolvimento biopsicossocial ao longo do tempo.

Alguns limites também se impuseram nessa revisão. Primeiramente, como forma de estabelecer um maior rigor nos critérios de inclusão/exclusão da pesquisa, deixou-se de fora revisões sistemáticas e narrativas, artigos técnicos, teses e dissertações, bem como capítulos de livros que poderiam oferecer um olhar mais abrangente e integral do estado da arte sobre o tema da resiliência e a população LGB. Além disso, outras bases de dados como Scopus, Web of Science, por exemplo, também poderiam ser incluídas, ampliando a compreensão do estado da arte acerca da temática aqui estudada. Ao mesmo tempo, a ampliação da busca pode contribuir para a consolidação do construto da resiliência na promoção de estratégias práticas destinadas a melhorar a saúde e bem-estar das minorias sexuais.

Gays e lésbicas continuam a manter suas relações num contexto marcado pela discriminação homofóbica e de desproteção de políticas públicas (Doyle \& Molix, 2015; Lira \& Morais, 2017). Certamente, ao identificar a resiliência nos diversos níveis ecológicos que as minorias sexuais estão envolvidas, ampliam-se as chances de delinear intervenções em uma variedade de contextos (na família, nos equipamentos de 
saúde, de educação, na assistência social), com o intuito de minimizar os riscos ou exposição às adversidades que acompanham a vida das minorias sexuais, aumentar os recursos propulsores do seu desenvolvimento, bem como fortalecer ou restaurar os seus sistemas adaptativos (Wright \& Masten, 2015).

\section{Referências}

Azevedo, R. S. (2010). Sobrecarga do cuidador informal da pessoa idosa frágil: Uma revisão sistemática (Dissertação de mestrado não publicada, Universidade Federal de Minas Gerais, Belo Horizonte, MG, Brasil).

*Bonanno, G. A., Moskowitz, J. T., Papa, A., \& Folkman, S. (2005). Resilience to loss in bereaved spouses, bereaved parents, and bereaved gay men. Journal of Personality and Social Psychology, 88(5), 827. doi: 10.1037/00223514.88.5.827

*Bowleg, L., Huang, J., Brooks, K., Black, A., \& Burkholder, G. (2003). Triple jeopardy and beyond: Multiple minority stress and resilience among black lesbians. Journal of Lesbian Studies, 7(4), 87-108. doi: 10.1300/J155v07n04_06

*Bruce, D., Harper, G. W., \& Bauermeister, J. A. (2015). Minority stress, positive identity development, and depressive symptoms: Implications for resilience among sexual minority male youth. Psychology of Sexual Orientation and Gender Diversity, 2(3), 287. doi: http://dx.doi. org/10.1037/sgd0000128

*Buttram, M. E., Surratt, H. L., \& Kurtz, S. P. (2014). Risk and protective factors associated with personal mastery among sexual minority African-American female sex workers. Journal of Gay \& Lesbian Social Services, 26(4), $407-$ 425. doi: 10.1080/10538720.2014.956242

Colpitts, E., \& Gahagan, J. (2016). The utility of resilience as a conceptual framework for understanding and measuring LGBTQ health. International Journal for Equity in Health, 15(1), 1. doi: 10.1186/s12939-0160349-1

*DiFulvio, G. T. (2011). Sexual minority youth, social connection and resilience: From personal struggle to collective identity. Social Science \& Medicine, 72(10), 1611-1617. doi: 10.1016/j. socscimed.2011.02.045
Doyle, D. M., \& Molix, L. (2015). Social stigma and sexual minorities' romantic relationship functioning: A meta-analytic review. Personality and Social Psychology Bulletin, 41(10), 1363-1381. doi: $10.1177 / 0146167215594592$

*Erhard, R. L., \& Ben-Ami, E. (2015). The schooling experience of lesbian, gay, and bisexual youth in lsrael: Falling below and rising above as a matter of social ecology. Journal of homosexuality, 63(2), 193-227. doi: $10.1080 / 00918369.2015 .1083778$

*Farr, R. H., Crain, E. E., Oakley, M. K., Cashen, K. K., \& Garber, K. J. (2016). Microaggressions, feelings of difference, and resilience among adopted children with sexual minority parents. Journal of Youth and Adolescence, 45(1), 85-104. doi: 10.1007/s10964-015-0353-6

*Figueroa, W. S., \& Zoccola, P. M. (2015). Individual differences of risk and resiliency in sexual minority health: The roles of stigma consciousness and psychological hardiness. Psychology of Sexual Orientation and Gender Diversity, 2(3), 329. doi: http://dx.doi.org/10.1037/sgd0000114

*Foster, K. A., Bowland, S. E., \& Vosler, A. N. (2015). All the pain along with all the joy: Spiritual resilience in lesbian and gay Christians. American Journal of Community Psychology, 55(1-2), 191-201. doi: 10.1007/s10464-015-9704-4

*Fredriksen-Goldsen, K. I., Emlet, C. A., Kim, H. J., Muraco, A., Erosheva, E. A., Goldsen, J., \& Hoy-Ellis, C. P. (2012). The physical and mental health of lesbian, gay male, and bisexual (LGB) older adults: The role of key health indicators and risk and protective factors. The Gerontologist, 53(4), 664-675. doi: 10.1093/geront/gns123

*Fredriksen-Goldsen, K. I., Kim, H. J., Shiu, C., Goldsen, J., \& Emlet, C. A. (2015). Successful aging among LGBT older adults: Physical and mental health-related quality of life by age group. The Gerontologist, 55(1), 154-168. doi: 10.1093/ geront/gnu081

*Gray, N. N., Mendelsohn, D. M., \& Omoto, A. M. (2015). Community connectedness, challenges, and resilience among gay Latino immigrants. American Journal of Community Psychology, 55(1-2), 202-214. doi: 10.1007/s10464-0149697-4

*Gwadz, M. V., Clatts, M. C., Yi, H., Leonard, N. R., Goldsamt, L., \& Lankenau, S. (2006). Resilience among young men who have sex with men in New York City. Sexuality Research and 
Social Policy Journal of NSRC, 3(1), 13-21. doi: $10.1525 /$ srsp.2006.3.1.13

*Harper, G. W., Bruce, D., Hosek, S. G., Fernandez, M. I., \& Rood, B. A. (2014). Resilience processes demonstrated by young gay and bisexual men living with hiv: Implications for intervention. AIDS Patient Care and STDs, 28(12), 666676. doi: 10.1525/srsp.2006.3.1.13

*Herrick, A. L., Stall, R., Chmiel, J. S., Guadamuz, T. E., Penniman, T., Shoptaw, S., ...Plankey, M. W. (2013). It gets better: Resolution of internalized homophobia over time and associations with positive health outcomes among MSM. AIDS and Behavior, 17(4), 1423-1430. doi: 10.1007/ s10461-012-0392-x

Hill, C. A., \& Gunderson, C. J. (2015). Resilience of lesbian, gay, and bisexual individuals in relation to social environment, personal characteristics, and emotion regulation strategies. Psychology of Sexual Orientation and Gender Diversity, 2(3), 232. doi: $10.1037 / \operatorname{sgd} 0000129$

*Kosciw, J. G., Palmer, N. A., \& Kull, R. M. (2015). Reflecting resiliency: Openness about sexual orientation and/or gender identity and its relationship to well-being and educational outcomes for LGBT students. American Journal of Community Psychology, 55(1-2), 167-178. doi: 10.1007/s10464-014-9642-6

*Kubicek, K., McNeeley, M., Holloway, I. W., Weiss, G., \& Kipke, M. D. (2013). "It's like our own little world": Resilience as a factor in participating in the ballroom community subculture. AIDS and Behavior, 17(4), 1524-1539. doi: 10.1007/s10461-012-0205-2

*Kurtz, S. P., Buttram, M. E., Surratt, H. L., \& Stall, R. D. (2012). Resilience, syndemic factors, and serosorting behaviors among HIVpositive and HIV-negative substance-using MSM. AIDS Education and Prevention: Official Publication of the International Society for AIDS Education, 24(3), 193. doi: 10.1521/ aeap.2012.24.3.193

Kwon, P. (2013). Resilience in lesbian, gay, and bisexual individuals. Personality and Social Psychology Review, 17(4), 371-383. doi: $10.1177 / 1088868313490248$

Lever, J. P., \& Valdez, N. E. G. (2010). Desarrollo de una escala de medicion de la residencia con mexicanos (RESI-M). Interdisciplinaria, 27(1), 7-23. Retrieved from http://www.redalyc.org/ pdf/180/18014748002.pdf
Liberati, A., Altman, D. G., Tetzlaff, J., Mulrow, C., Gotzsche, P. C., Ioannidis, J. P. A., ...Moher, D. (2009). The PRISMA statement for reporting systematic reviews and meta-analyses of studies that evaluate health care interventions: Explanation and elaboration. Annals of Internal Medicine, 151(4), 65-94. doi: https://doi.org/10.1136/ bmj.b2700

Lira, A. N., \& Morais, N. A. (2017). Resilience in lesbian, gay and bisexual (LGB) populations: An integrative literature review. Sexuality Research and Social Policy. doi: 10.1007/s13178-0170285-x

*Livingston, N. A., Heck, N. C., Flentje, A., Gleason, H., Oost, K. M., \& Cochran, B. N. (2015). Sexual minority stress and suicide risk: Identifying resilience through personality profile analysis. Psychology of Sexual Orientation and Gender Diversity, 2(3), 321. doi: http://dx.doi. org/10.1037/sgd0000116

Luthar, S. S., Cicchetti, D., \& Becker, B. (2000). The construct of resilience: A critical evaluation and guidelines for future work. Child Development, 71, 543-62. Retrieved from http://www.ncbi. nlm.nih.gov/pubmed/10953923

Lyons, A. (2015). Resilience in lesbians and gay men: A review and key findings from a nationwide Australian survey. Internacional Review of Psychiatry, 29, 1-9. doi: 10.3109/09540261.2015.1051517

*Mason, T. B., Lewis, R. J., Winstead, B. A., \& Derlega, V. J. (2015). External and internalized heterosexism among sexual minority women: The moderating roles of social constraints and collective self-esteem. Psychology of Sexual Orientation and Gender Diversity, 2(3), 313320. doi: http://dx.doi.org/10.1037/sgd0000115

Masten, A. S. (2015). Ordinary magic. Resilience in development. New York: Guilford Press.

Masten, A. S., \& Monn, A. R. (2015). Child and family resilience: A call for integrated science, practice and professional training. Family Relations, 64, 5-21. doi: 10.1111/fare.12103

*Mereish, E., \& Poteat, V. P. (2015). Effects of heterosexuals' direct and extended friendships with sexual minorities on their attitudes and behaviors: Intergroup anxiety and attitude strength as mediators and moderators. Journal of Applied Social Psychology, 45(3), 147-157. doi: http:// dx.doi.org/10.1037/sgd0000121 
Meyer, I. H. (2015). Resilience in the study of minority stress and health of sexual and gender minorities. Psychology of Sexual Orientation and Gender Diversity, 2(3), 213-219. doi: http:// dx.doi.org/10.1037/sgd0000132

*Mustanski, B., Newcomb, M. E., \& Garofalo, R. (2011). Mental health of lesbian, gay, and bisexual youths: A developmental resiliency perspective. Journal of Gay \& Lesbian Social Services, 23(2), 204-225. doi: 10.1080/10538720.2 011.561474

*Peterson, J. L., Bakeman, R., Sullivan, P., Millett, G., Rosenberg, E., Salazar, L., ...Frew, P. (2014). Social discrimination and resiliency are not associated with differences in prevalent HIV infection in black and white men who have sex with men. Journal of Acquired Immune Deficiency Syndromes, 66(5), 538. doi: 10.1097/ QAI.0000000000000203

*Poteat, V. P., Mereish, E. H., DiGiovanni, C. D., \& Koenig, B. W. (2011). The effects of general and homophobic victimization on adolescents' psychosocial and educational concerns: The importance of intersecting identities and parent support. Journal of Counseling Psychology, 58(4), 597. doi: 10.1037/a0025095

*Reisner, S. L., Biello, K., Perry, N. S., Gamarel, K. E., \& Mimiaga, M. J. (2014). A compensatory model of risk and resilience applied to adolescent sexual orientation disparities in nonsuicidal self-injury and suicide attempts. American Journal of Orthopsychiatry, 84(5), 545. doi: 10.1037/ ort0000008

*Rodríguez, M. D. C. F., \& Calle, F. V. (2013). En torno al rechazo, la salud mental y la resiliencia en un grupo de jóvenes universitarios gays, lesbianas y bisexuales. Revista Griot, 6(1), 44. Retrieved from http://www.ncbi.nlm.nih.gov/pmc/ articles/PMC4318519/

*Russell, G. M., \& Richards, J. A. (2003). Stressor and resilience factors for lesbians, gay men, and bisexuals confronting antigay politics. American Journal of Community Psychology, 31(3-4), 313-328. doi: 10.1023/A:1023919022811

*Shilo, G., Antebi, N., \& Mor, Z. (2015). Individual and community resilience factors among lesbian, gay, bisexual, queer and questioning youth and adults in israel. American Journal of Community Psychology, 55(1-2), 215-227. doi: 10.1007/ s10464-014-9693-8

*Sung, M. R., Szymanski, D. M., \& Henrichs-Beck, C. (2015). Challenges, coping, and benefits of being an Asian American lesbian or bisexual woman. Psychology of Sexual Orientation and Gender Diversity, 2(1), 52. doi: http://dx.doi. org/10.1037/sgd0000085

Ungar, M. (2015). Resilience and culture: The diversity of protective processes and positive adaptation. In L. C. Theron, L. Liebenberg, \& M. Ungar, Youth resilience and culture (pp. 37-48). Dordrecht, Netherlands: Springer. doi: 10.1007/978-94-017-9415-2 3.

Wagnild, G., \& Young, H. (1993). Development and psychometric. Journal of Nursing Measurement, 1(2), 165-178.

*Walker, J. N. J., \& Longmire-Avital, B. (2013). The impact of religious faith and internalized homonegativity on resiliency for black lesbian, gay, and bisexual emerging adults. Developmental Psychology, 49(9), 1723. doi: 10.1037/a0031059

Wright, M. O. D., \& Masten, A. S. (2015). Pathways to resilience in context. In L. C. Theron, L. Liebenberg, \& M. Ungar, Youth resilience and culture (pp. 3-22). Dordrecht, Netherlands: Springer.

Yunes, M. A. M. (2003). Psicologia positiva e resiliência: O foco no indivíduo e na família. Psicologia em Estudo, 8(1), 80-95.

*Zimmerman, L., Darnell, D. A., Rhew, I. C., Lee, C. M., \& Kaysen, D. (2015). Resilience in community: A social ecological development model for young adult sexual minority women. American Journal of Community Psychology, 55(1-2), 179-190. doi: 10.1007/s10464-015-9702-6

Recebido: 03/04/2017

$1^{a}$ revisão: $29 / 05 / 2017$

$2^{a}$ revisão: $13 / 08 / 2017$

Aceite final: 25/08/2017 